\title{
First evidence of scale drop disease virus in farmed Asian seabass (Lates calcarifer) in Malaysia
}

\begin{abstract}
An unknown disease event in marine cage-cultured Asian seabass (Lates calcarifer) was observed in Malaysia in September 2019, which affected adult-sized fish and resulted in significant mortality. Affected fish showed gross signs resembling scale drop disease such as lethargy, darkened dorsal part of the body, easily detached scales, severe scale loss, reddening and hemorrhage at the ventral part of the body, and tendency to swim at the water surface. Histopathological examinations revealed abnormal changes in multiple internal organs including vacuolated cytoplasm hepatocytes, necrotic kidney tubules, atrophied glomeruli, blood congestion and hemorrhage in the spleen and brain, as well as penetration of lymphocytic inflammatory cells. Notably, basophilic hypertrophied cells and intracytoplasmic inclusion bodies were occasionally observed in the liver, indicating suspected viral aetiology. Transmission electron microscopy (TEM) revealed the presence of multiple viral particles in the cytoplasm of the infected cells. Subsequently, PCR and sequence analyses revealed that all affected fish were positive to scale drop disease virus (SDDV), which was absent from the clinically healthy fish. Phylogeny analysis based on complete ORF $060 \mathrm{~L}$ (encoding major capsid protein) revealed high similarity between the SDDV from Malaysia, Thailand and Singapore. This study provides first evidence of SDDV infection in cultured Asian seabass in Malaysia. We thus recommend that active surveillance should be prioritized for concentrated Asian seabass farming areas in Malaysia to prevent spread of the disease nation-wide.
\end{abstract}

Keyword: Scale drop disease virus; Lates calcarifer; Asian seabass; Malaysia 